\title{
Triangular Sequences, Combinatorial Recurrences and Linear Difference Equations
}

\author{
A.M. Encinas and M.J. Jiménez \\ Matemàtiques, UPC, BarcelonaTech, Spain
}

\begin{abstract}
In this work we introduce the triangular double sequences of arbitrary order given by linear recurrences, that generalize some well-known recurrences that appear in enumerative combinatorics. In particular, we focussed on triangular sequences generated by two double sequences and establish their relation with the solution of linear three-term recurrences. We show through some simple examples how these triangular sequences appear as essential components in the expression of some classical orthogonal polynomials and combinatorial numbers.
\end{abstract}

Keywords: Combinatorial Identities, Triangular matrices, Linear Difference Equations, Three-term Recurrences, Orthogonal Polynomials

2010 MSC: 05A10, 05A19, 11B37, 11B65, 11B73, 39A06

\section{Introduction and Preliminaries}

The motivation of the main results in this work arises from the analysis of the second order linear difference equations developed by the authors in [1. Although there, the general framework and methods followed the guidelines of the linear differential equations, the expression of their solutions had an strong combinatorial flavour. Deepening in this algebraic structure, with the aim of finding more manageable expressions of the solutions, we found that they could be expressed through double sequences, or infinite matrices, determined by linear recurrences. It results that the structure of these recurrences is the same than what appears in the so-called Galton arrays, see [2, but the new ones have more null entries. The generalization of these recurrences leaded us to define triangular arrays of order greater than one, the Galton arrays corresponding precisely with those of order 1 . We show that some triangular sequences, that we name row-generated are those related with linear three-term recurrences, being the order of the difference equation, the order of the triangular array.

In this paper, we present a systematic way for obtaining triangular sequences of any order from linear recurrences. Identifying each triangular sequence with an infinite matrix, our methodology consists in calculate each column from the preceding ones by solving difference equations of order 1. Particularizing this technique to row-generated triangular sequences, our results imply that any irreducible second order 
linear difference equation, or more generally some difference equations of any order but determined by a three-term recurrence, can be solved by means of a sequence of first order linear difference equations. To our understanding, this represent a huge distinction between the treatment of differential and difference linear equations.

Throughout the paper, $\mathbb{C}, \mathbb{R}$ and $\mathbb{Q}$ denote the fields of complex, real and rational numbers, respectively, $\mathbb{Z}$ the ring of integers, $\mathbb{N}$ the set of non-negative integers and $\mathbb{N}^{*}$ the set of positive integers; that is, $\mathbb{N}^{*}=\mathbb{N} \backslash\{0\}$. Moreover, $(\mathbb{A},+, \cdot)$ denotes a commutative ring with identity and $\mathbb{A}^{*}$ its group of units. As usual, the additive and the multiplicative identity of $\mathbb{A}$ are denoted by 0 and 1 respectively, and given $25 a \in \mathbb{A}^{*}$, its inverse is denoted by $a^{-1}$. Given $r \in \mathbb{A}$, we always assume that $r^{0}=1$, even when $r=0$, and in addition, the usual convention that empty sums and empty products are defined as 0 and 1 , respectively.

A sequence of elements of $\mathbb{A}$ is a function $a: \mathbb{N} \longrightarrow \mathbb{A}$. We denote by $\ell(\mathbb{A})$ the space of all sequences of elements of $\mathbb{A}$ and by $\ell\left(\mathbb{A}^{*}\right) \subset \ell(\mathbb{A})$ the subset of the sequences $a \in \ell(\mathbb{A})$ such that $a(k) \in \mathbb{A}^{*}$ for all $k \in \mathbb{N}$. When necessary, we consider each $a \in \ell(\mathbb{A})$ extended by 0 to $\mathbb{Z}$; that is, we assume that $a(k)=0$ when so $k \in \mathbb{Z} \backslash \mathbb{N}$.

Clearly $(\ell(\mathbb{A}),+, \cdot)$ is a commutative ring with identity, where the sum and the product of $a, b \in \ell(\mathbb{A})$ are defined as $(a+b)(k)=a(k)+b(k)$ and $(a b)(k)=(a \cdot b)(k)=a(k) \cdot b(k)$ for any $k \in \mathbb{N}$. Therefore, the additive identity in $\ell(\mathbb{A})$ is the null sequence, whereas the multiplicative identity of $\ell(\mathbb{A})$ is the constant sequence whose entries are all equals 1 . Moreover, identifying $a \in \mathbb{A}$ with the constant sequence whose value at any $k \in \mathbb{N}$ is $a$, we get that $\mathbb{A} \subset \ell(\mathbb{A})$ is a subring of $\ell(\mathbb{A})$. Throughout the paper, we use this identification and hence if $a \in \mathbb{A}$ we denote the corresponding constant sequence also by $a$. With this convention in mind, 0 is the additive identity in $\ell(\mathbb{A})$, whereas the multiplicative identity of $\ell(\mathbb{A})$ is 1 . Clearly, $\ell^{*}(\mathbb{A})=\ell\left(\mathbb{A}^{*}\right)$ and moreover if $a \in \ell\left(\mathbb{A}^{*}\right)$, then its multiplicative inverse is denoted either by $a^{-1}$ or by $\frac{1}{a}$.

A double sequence of elements of $\mathbb{A}$ is a function $h: \mathbb{N} \times \mathbb{N} \longrightarrow \mathbb{A}$ and we denote by $\ell_{2}(\mathbb{A})$ the space of all double sequences of elements of $\mathbb{A}$ and by $\ell_{2}\left(\mathbb{A}^{*}\right) \subset \ell_{2}(\mathbb{A})$ the subset of the double sequences $h \in \ell_{2}(\mathbb{A})$ such that $h(k, m) \in \mathbb{A}^{*}$ for all $k, m \in \mathbb{N}$. When necessary we consider each $h \in \ell_{2}(\mathbb{A})$ extended by 0 to $\mathbb{Z} \times \mathbb{Z}$; that is, we assume that $h(k, m)=0$ when either $k \in \mathbb{Z} \backslash \mathbb{N}$ or $m \in \mathbb{Z} \backslash \mathbb{N}$.

Newly $\left(\ell_{2}(\mathbb{A}),+, \cdot\right)$ is a commutative ring with identity, where the sum and the product of $g, h \in \ell_{2}(\mathbb{A})$ are defined as $(g+h)(k, m)=g(k, m)+h(k, m)$ and $(g \cdot h)(k, m)=g(k, m) \cdot h(k, m)$ for any $k \in \mathbb{N}$. Therefore, the additive identity in $\ell_{2}(\mathbb{A})$ is the null sequence, whereas the multiplicative identity of $\ell_{2}(\mathbb{A})$ is the constant double sequence whose entries are all equals 1 . Moreover, $\ell_{2}^{*}(\mathbb{A})=\ell_{2}\left(\mathbb{A}^{*}\right)$ and when $h \in \ell_{2}\left(\mathbb{A}^{*}\right)$, then its multiplicative inverse is denoted by $h^{-1}$.

Notice that if, in a natural way, we identify $\ell_{2}(\mathbb{A})$ with the space of infinite matrices whose entries are elements of $\mathbb{A}$, indexing rows and columns from 0 , then the ordinary sum and product in $\ell_{2}(\mathbb{A})$ correspond 
to the entry-wise sum and product, respectively. Recall that the entry-wise product is also known as the Hadamard product of the matrices.

Given $a, b \in \ell(\mathbb{A})$, we denote by $a \otimes b \in \ell_{2}(\mathbb{A})$ the double sequence defined as

$$
(a \otimes b)(k, m)=a(k) b(m), \text { for any } k, m \in \mathbb{N}
$$

It is clear that $h \in \ell_{2}(\mathbb{A})$ does not depend on its first variable iff $h=1 \otimes a$, respectively does not depend on its second variable iff $h=a \otimes 1$

Given $a \in \ell(\mathbb{A})$ we also consider the sequence $a^{\pi} \in \ell(\mathbb{A})$ defined for any $k \in \mathbb{N}$ as

$$
a^{\pi}(k)=\prod_{s=0}^{k-1} a(s)
$$

which implies that $a^{\pi}(0)=1$ and $a^{\pi}(1)=a(0)$. Moreover, we also define $a^{\pi}(k)=1$ for $k \in \mathbb{Z} \backslash \mathbb{N}$. In particular, when $\mathbb{A}=\mathbb{R}$ and $a(k)=k+x$, where $x \in \mathbb{R}$, the sequence $a^{\pi}$ is usually known as factorial function and denoted by $(x)$, see for instance [3]. Therefore, $a^{\pi}(k)=(x)_{k}$, for any $k \in \mathbb{N}$ and moreover, when $x>0$, we have $(x)_{k}=\frac{\Gamma(k+x)}{\Gamma(x)}$, for any $k \in \mathbb{N}$, where $\Gamma$ denotes the Gamma function.

Given $a, c \in \ell(\mathbb{A})$, for any $n \in \mathbb{N}^{*}$ we denote by $a \star_{n} c$ the sequence defined for any $k \in \mathbb{N}$ as

$$
\left(a \star_{n} c\right)(k)=c(k) \prod_{s=k+2-n}^{k} \hat{a}(s), \quad \text { where } \hat{a}(k)=a(k) \text { if } k \in \mathbb{N} \text { and } \hat{a}(k)=1 \text { if } k \in \mathbb{Z} \backslash \mathbb{N} .
$$

Observe that $a \star_{1} c=c$, whereas $a \star_{2} c=a c$. Sequences of the type $a \star_{n} c$ will appear in the last section related with the solution of three-term recurrences.

Since homogeneous linear difference equations of order 1 appear ubiquitously in this work, we conclude this section with a basic result about them that will be systematically used throughout the rest of the paper. Specifically, given $a, b \in \ell(\mathbb{A})$, for any $m \in \mathbb{N}$ we call solution of the initial value problem

$$
z(m)=b(m), \quad z(k)=a(k-1) z(k-1)+b(k), \quad k \geq m+1,
$$

any sequence $z \in \ell(\mathbb{A})$ satisfying the above identities for $k \geq m$ and moreover we always assume that $z(j)=0$ for $0 \leq j<m$. Clearly, the initial value problem (4) is equivalent to the recurrence

$$
z(k)=a(k-1) z(k-1)+b(k), \quad k \geq m, \quad z(j)=0, \quad 0 \leq j<m .
$$

The main result about the above recurrence is the following one. Its proof is reduced to a simple verification, see also [1, Proposition 3.1] for a more general expression.

Proposition 1.1. Given $m \in \mathbb{N}$, the recurrence (5) has a unique solution that is determined by

$$
z(k)=\sum_{s=m}^{k}\left(\prod_{j=s}^{k-1} a(j)\right) b(s), \quad k \in \mathbb{N} .
$$


In particular, when $a \in \ell\left(\mathbb{A}^{*}\right)$, then

$$
z(k)=a^{\pi}(k) \sum_{s=m}^{k} a^{\pi}(s)^{-1} b(s), \quad k \geq m .
$$

\section{Triangular sequences}

In this section we study an special class of double sequences or infinite matrices whose entries are elements of $\mathbb{A}$. Specifically we work with some special classes of infinite lower triangular matrices.

With the same spirit than in [2], given $n \in \mathbb{N}^{*}$, we call triangular sequence of order $n$ in $\mathbb{A}$ to any double sequence $T \in \ell_{2}(\mathbb{A})$ satisfying that

$$
T(k, m)=0 \quad \text { when } m \geq 1 \text { and } 0 \leq k<m n
$$

and denote by $\Lambda_{n}(\mathbb{A})$ the set of triangular sequences of order $n$. Equivalently, $T \in \ell_{2}(\mathbb{A})$ is triangular sequence of order $n$ iff $T(k, m)=0$ when $m>\left\lfloor\frac{k}{n}\right\rfloor$. Moreover, we also have that $T(0, m)=0$ for $m \geq 1$.

For the sake of completeness, we also consider $n=0$ and hence $\Lambda_{0}(\mathbb{A})=\ell_{2}(\mathbb{A})$. In any case, $\Lambda_{n}(\mathbb{A})$ is a vector subspace of $\ell_{2}(\mathbb{A})$ and moreover $\Lambda_{n_{2}}(\mathbb{A}) \subset \Lambda_{n_{1}}(\mathbb{A})$ when $0 \leq n_{1} \leq n_{2}$.

Taken into account the identification between double sequences and infinite matrices, it appears that $\Lambda_{1}(\mathbb{A})$ is identified with the space of lower triangular matrices. In addition, when $n \geq 2, \Lambda_{n}(\mathbb{A})$ can be identified with lower triangular matrices that in each column has more null entries, specifically in column $m$ not only the first $m$ entries are null, remember that we are indexing rows and columns from 0 , but all the first $m n$ ones; that is, there are $m(n-1)$ more null entries.

The following basic result shows how to obtain many triangular sequences by superposing double sequences. It provides us with useful and easy identities that we consider throughout this work. For the sake of a clean writing, hereinafter we always assume that $s_{0}=0$.

Consider a family of double sequences $\Phi \equiv\left\{\Phi_{m}\right\}_{m \in \mathbb{N}^{*}} \subset \ell_{2}(\mathbb{A})$. For any $n \in \mathbb{N}^{*}$, we call kernel of order $n$ on $\mathbb{A}$ with core $\Phi$, the double sequence $K_{n}(\Phi) \in \ell_{2}(\mathbb{A})$ defined from the recurrence

$$
\begin{cases}K_{n}(\Phi)(s, 0)=1, & s \geq 0 \\ K_{n}(\Phi)(s, 1)=\Phi_{1}\left(s_{0}, s-n\right), & s \geq 0 \\ K_{n}(\Phi)(s, m)=\sum_{s_{m-1}=(m-1) n}^{s-n} \Phi_{m}\left(s_{m-1}, s-n\right) K_{n}(\Phi)\left(s_{m-1}, m-1\right), & s \geq 0, m \geq 2 .\end{cases}
$$

In addition, we call kernel on $\mathbb{A}$ with core $\Phi$, the familiy of kernels $\mathscr{K}(\Phi) \equiv\left\{K_{n}(\Phi)\right\}_{n \in \mathbb{N}^{*}}$.

Notice that for any $n, m \in \mathbb{N}^{*}$ and for any $s \in \mathbb{N}, K_{n}(\Phi)(s, m)$ only takes into account the values of the core sequences $\Phi_{m}$ on the set $\left\{\left(s_{m-1}, s\right):(m-1) n \leq s_{m-1} \leq s-n\right\}$ and it is null otherwise. Therefore, for any $m \geq 1$ for $K_{n}(\Phi)(s, m)$ takes a non null value it is necessary that $s \geq m n$, which implies that 
$K_{n}(\Phi) \in \Lambda_{n}(\mathbb{A})$ for any $n \in \mathbb{N}^{*}$. The following result provides us with useful expressions for the kernel of order $n \in \mathbb{N}^{*}$ on $\mathbb{A}$ with core $\Phi$.

Lemma 2.1. Given $\Phi \equiv\left\{\Phi_{m}\right\}_{m \in \mathbb{N}^{*}} \subset \ell_{2}(\mathbb{A})$, then $K_{n}(\Phi) \in \Lambda_{n}(\mathbb{A})$ for any $n \in \mathbb{N}^{*}$. Moreover, for any $m \geq 2$ we have

$$
K_{n}(\Phi)\left(s_{m}+m(n-1), m\right)=\sum_{1 \leq s_{1}<\cdots<s_{m-1}<s_{m}} \prod_{i=0}^{m-1} \Phi_{i+1}\left(s_{i}+i(n-1), s_{i+1}+i(n-1)-1\right), \quad s_{m} \geq m .
$$

In particular, $K_{n}(\Phi)(m n, m)=\prod_{i=0}^{m-1} \Phi_{i+1}(i n, i n)$, for any $m \in \mathbb{N}$.

Observe that if we consider $0 \leq s_{m}<m$ in the first identity, then $K_{n}(\Phi)\left(s_{m}+m(n-1), m\right)=0$, since in this case the sum on the right is empty. With this consideration, the special case of the above result when $n=1$, that corresponds to lower triangular matrices, reads as

$$
K_{1}(\Phi)\left(s_{m}, m\right)=\sum_{1 \leq s_{1}<\cdots<s_{m-1}<s_{m}} \prod_{i=0}^{m-1} \Phi_{i+1}\left(s_{i}, s_{i+1}-1\right), \text { for any } s_{m} \in \mathbb{N} .
$$

In this paper we are focused on an specific class of kernels that are generated by two double sequences. Specifically, given $g, h \in \ell_{2}(\mathbb{A})$, we call the core generated by $g$ and $h$ to $\Phi[g, h]=\left\{\Phi_{m}[g, h]\right\}_{m \in \mathbb{N}^{*}}$, where for $m \geq 1$

$$
\Phi_{m}[g, h](s, k)=h(k, m-1) \prod_{j=s}^{k-1} g(j, m-1), \quad s, k \in \mathbb{N} .
$$

and kernel on $\mathbb{A}$ generated by $g$ and $h$ to the kernel $\mathscr{K}[g, h] \equiv\left\{K_{n}[g, h]\right\}_{n \in \mathbb{N}^{*}}$ which core is $\Phi[g, h]$. Therefore, applying Lemma 2.1 we obtain the expression for this kernel.

Proposition 2.2. Given $g, h \in \ell_{2}(\mathbb{A})$, then for any $n \in \mathbb{N}^{*}$, we have the following identities:

$$
\begin{aligned}
K_{n}[g, h](s, 0) & =1, & & s \geq 0 \\
K_{n}[g, h]\left(s_{1}+n, 1\right) & =h\left(s_{1}, 0\right) \prod_{j=0}^{s_{1}-1} g(j, 0), & & s_{1} \geq 0,
\end{aligned}
$$

and for any $s_{m} \geq m \geq 2$,

$$
K_{n}[g, h]\left(s_{m}+m(n-1), m\right)=\sum_{1 \leq s_{1}<\cdots<s_{m-1}<s_{m}} \prod_{i=0}^{m-1} h\left(s_{i+1}+i(n-1)-1, i\right) \prod_{j=s_{i}}^{s_{i+1}-2} g(j+i(n-1), i) .
$$

In particular, $K_{n}[g, h](m n, m)=\prod_{i=0}^{m-1} h(i n, i)$, for any $m \in \mathbb{N}$. 
Newly, we specify the special case of the above Proposition when $n=1$ :

$$
K_{1}[g, h]\left(s_{m}, m\right)=\sum_{1 \leq s_{1}<\cdots<s_{m-1}<s_{m}} \prod_{i=0}^{m-1} h\left(s_{i+1}-1, i\right) \prod_{j=s_{i}}^{s_{i+1}-2} g(j, i), \text { for any } s_{m} \in \mathbb{N} .
$$

Of course not any core, and hence any kernel, is generated by a pair of double sequences. For instance, if we define Harmonic kernel $K(\Theta)$ as the kernel on $\mathbb{Q}$, which core $\Theta \equiv\left\{\Theta_{m}\right\}_{m \in \mathbb{N}^{*}}$ is given by

$$
\Theta_{m}(s, k)=\frac{1}{s+1}, \text { for any } m \geq 1
$$

it is easy to prove that it is not generated for any pair of double sequences. For this kernel, given $n \in \mathbb{N}^{*}$ we have $K_{n}(\Theta)\left(s_{1}+n, 1\right)=\frac{1}{s+1}$, for $s \geq 0$ and moreover,

$$
K_{n}(\Theta)\left(s_{m}+m(n-1), m\right)=\sum_{1 \leq s_{1}<\cdots<s_{m-1}<s_{m}} \prod_{i=0}^{m-1} \frac{1}{s_{i}+i(n-1)}, \text { for } s_{m} \geq m \geq 2 .
$$

In particular,

$$
K_{1}(\Theta)\left(s_{1}, 1\right)=\frac{1}{s_{1}} \text { for } s_{1} \geq 1 \text { and } K_{1}(\Theta)\left(s_{m}, m\right)=\sum_{1 \leq s_{1}<\cdots<s_{m-1}<s_{m}} \frac{1}{s_{1} \cdots s_{m}}, \quad \text { for } s_{m} \geq m \geq 2 .
$$

The harmonic kernel of order 1 is closely related with the Generalized harmonic numbers that were defined by I.M. Gessel in [4], see also [5] 6], as

$$
H(k, 0)=1, \quad k \geq 0 \text { and } H(k, m)=\sum_{1 \leq s_{1}<\cdots<s_{m} \leq k} \frac{1}{s_{1} \cdots s_{m}}, \quad k \geq m \geq 1 .
$$

Notice that $H(k, k)=\frac{1}{k !}, k \geq 0$ and $H(k, 1)=\sum_{s=1}^{k} \frac{1}{s}, k \geq 1$, is the $k$-th harmonic number. Moreover as double sequence it is clear that $H \in \Lambda_{1}(\mathbb{Q})$ and for any $k \geq m \geq 1$, the Generalized harmonic number $H(k, m)$, is obtained summing the values of the Harmonic kernel of order 1 between $m$ and $k$ :

$$
H(k, m)=\sum_{s=m}^{k} K_{1}(\Theta)(s, m), \quad k \geq m
$$

Our propose is to show that as in the above example, many triangular sequences on $\mathbb{A}$ can be obtained in this way, as superposition of suitable kernels on $\mathbb{A}$. Prior to tackle the general question, we list some 90 well-known examples of triangular sequences in $\Lambda_{1}(\mathbb{R})$ that play a main role in the general treatment of this kind of sequences, see [7, 2, , 8]. If $T$ is anyone of this triangular sequences, $T(0,0)=1$ and the other entries are defined by means of a linear recurrence.

(i) Binomial coefficients, $B(k, m)=B(k-1, m)+B(k-1, m-1)$.

(ii) Signless Stirling numbers of first kind, $\left[\begin{array}{c}k \\ m\end{array}\right]=k\left[\begin{array}{c}k-1 \\ m\end{array}\right]+\left[\begin{array}{c}k-1 \\ m-1\end{array}\right]$. 
(iii) Stirling numbers of second kind, $\left\{\begin{array}{l}k \\ m\end{array}\right\}=m\left\{\begin{array}{c}k-1 \\ m\end{array}\right\}+\left\{\begin{array}{c}k-1 \\ m-1\end{array}\right\}$.

(iv) Generalized harmonic numbers, $H(k, m)=H(k-1, m)+\frac{1}{k} H(k-1, m-1)$

We remark that, in fact, the sequences (i) to (iii) belong to $\ell_{2}(\mathbb{Z}) \subset \ell_{2}(\mathbb{A})$.

Following the above examples, the triangular sequences we consider here are defined by linear recurrence relations. Specifically, we focus on those double sequences $T \in \ell_{2}(\mathbb{A})$ satisfying

$$
\left\{\begin{aligned}
T(0,0) & =t_{0}, \\
T(k, m) & =g(k-1, m) T(k-1, m)+h(k-n, m-1) T(k-n, m-1), \quad k+m \geq 1 .
\end{aligned}\right.
$$

where $n \in \mathbb{N}^{*}, t_{0} \in \mathbb{A}$ and and $g, h \in \ell_{2}(\mathbb{A})$.

Notice that if $T$ satisfies (11), then $T(0, m)=0$ for $m \geq 1$, since $g$ and $h$ are null when anyone of their arguments does not belong to $\mathbb{N}$. Observe also that the triangular sequences of order $n=1$ from (i) to (iii) above mentioned, correspond to the recurrence (11) where $t_{0}=1$ and $h=1$ in all cases and $g=1$, $g(k, m)=k$ or $g(k, m)=m$, respectively. In addition, the Generalized harmonic numbers corresponds to the recurrence 111) when $t_{0}=1, g=1$ and $h(k, m)=\frac{1}{k+1}$.

The class of triangular sequences of order $n=1$ determined by the recurrence (11) are well-known and appears in the mathematical literature under different names, mainly with the aim of solving a question raised by R. Graham, D. Knuth and O. Patashnik in [9, Problem 6.94]. For instance, E. Neuwirth called them pure Galton arrays in [2, whereas P. Théorêt, see [10, 11, 12, named them hyperbinomial.

Notice that recurrences (i), (ii) and (iii) correspond to consider $g$ and $h$ as first order polynomials; that is, affine functions on $k$ and $m$. This is precisely the problem raised by Graham, Knuth and Patashnik that have been recently solved by J.F. Barbero, J. Salas and E.J.S. Villaseñor in [7 by means of the so-called bivariate exponential generating functions. In fact, this kind of technique comes from the above mentioned works by P. Théorêt and references therein. In these works, each hyperbinomial; that is, triangular sequences satisfying a recurrence of type (11), in which $g$ and $h$ are polynomials of the first degree is called special hyperbinomial. On the other hand, M. Z. Spivey solved the recurrences (11), see [8, for some cases of special hyperbinomials by using finite differences. Precisely, we use this last kind of techniques to prove our main result about linear recurrences. Observe that identifying $T$ with an infinite matrix, the recurrence (11) says that each column of $T$ can be obtained from the previous one throughout a first order linear equation.

The main result in this section is to obtain the expression of the double sequences satisfying the recurrence (11) in a closed form involving only the data $t_{0}$ and $g, h \in \ell_{2}(\mathbb{A})$. In fact, we prove that as in the case of the Generalized harmonic numbers, these double sequences can be determined as a suitable combination of the kernel generated by $g$ and $h$. 
Theorem 2.3. Given $t_{0} \in \mathbb{A}$ and $g, h \in \ell_{2}(\mathbb{A})$, for any $n \in \mathbb{N}^{*}$ there exists a unique double sequence $T$ satisfying (11). Moreover $T \in \Lambda_{n}(\mathbb{A})$ and for any $k \geq 0$ it is determined by the identities

$$
T(k, 0)=t_{0} \prod_{j=0}^{k-1} g(j, 0) \quad \text { and } \quad T(k, m)=t_{0} \sum_{s=m n}^{k}\left(\prod_{j=s}^{k-1} g(j, m)\right) K_{n}[g, h](s, m), \quad m \geq 1 .
$$

More explicitly, for any $k \geq m \geq 1$ we have that

$$
\begin{aligned}
T(k+m(n-1), m)=t_{0} \sum_{1 \leq s_{1}<\cdots<s_{m} \leq k} & \left(\prod_{i=0}^{m-1} h\left(s_{i+1}-1+i(n-1), i\right)\right) \\
& \times\left(\prod_{j=s_{m}}^{k-1} g(j+m(n-1), m)\right)\left(\prod_{i=0}^{m-1} \prod_{j=s_{i}}^{s_{i+1}-2} g(j+i(n-1), i)\right)
\end{aligned}
$$

Proof. First, notice that if the result is true then $T(m n, m)=t_{0} K_{n}[g, h](m n, m)=t_{0} \prod_{i=0}^{m-1} h(i n, i)$, for any $m \in \mathbb{N}$.

We always take into account that all double sequences vanish when one of the two arguments lies on the negative integers. We first prove that $T \in \Lambda_{n}(\mathbb{A})$. If $m=1$, from recurrence (11) we have

$$
T(k, 1)=g(k-1,1) T(k-1,1), \quad 0 \leq k<n
$$

which implies that $T(k, 1)=\left(\prod_{j=0}^{k-1} g(j, 1)\right) T(0,1)=0$, since $T(0,1)=0$. Assume that for $m \geq 1$ we have that $T(k, m)=0$ when $0 \leq k<n m$.

If $0 \leq k<n(m+1)$, then $k-n<n m$ and hence $T(k, m+1)=g(k-1, m+1) T(k-1, m+1)$, which implies that $T(k, m+1)=\left(\prod_{j=0}^{k-1} g(j, m+1)\right) T(0, m+1)=0$, since $T(0, m+1)=0$. Therefore, when $m \geq 1$, then $T(k, m)=0$ for any $0 \leq k<n m$; that is, $T \in \Lambda_{n}(\mathbb{A})$.

Since $T$ is triangular of order $n$, then for any $m \in \mathbb{N}$ we have that $T(n m-1, m)=0$ and hence, from the Identity 11,

$$
T(n m, m)=h(n(m-1), m-1) T(n(m-1), m-1), m \geq 1, T(0,0)=t_{0},
$$

which implies that $T(n m, m)=t_{0} \prod_{j=0}^{m-1} h(n j, j)$, for any $m \geq 1$. Clearly, the identity also holds when $m=0$, since the value of an empty product equals to 1 .

On the other hand, taking $m=0$ in the recurrence [11, we obtain that

$$
T(k, 0)=g(k-1,0) T(k-1,0), \quad k \geq 1 ; \quad T(0,0)=t_{0}
$$

and hence $T(k, 0)=t_{0} \prod_{j=0}^{k-1} g(j, 0)$, for any $k \geq 0$.

Fixed $m \in \mathbb{N}^{*}$, we consider the sequences $z, a, b \in \ell(\mathbb{A})$ defined for any $k \in \mathbb{N}$ as

$$
z(k)=T(k, m), \quad a(k)=g(k, m) \text { and } b(k)=h(k-n, m-1) T(k-n, m-1) .
$$


Therefore, we can re--write the recurrence (11) as the following initial value problem for a first order linear recurrence equation

$$
z(k)=a(k-1) z(k-1)+b(k), \quad z(n m)=b(n m) ; \quad k \geq m n+1 .
$$

Applying Proposition 1.1, we obtain that

$$
T(k, m)=\sum_{s=m n}^{k}\left(\prod_{j=s}^{k-1} a(j)\right) b(s)=\sum_{s=m n}^{k}\left(\prod_{j=s}^{k-1} g(j, m)\right) h(s-n, m-1) T(s-n, m-1)
$$

In fact, taking into account the convention about empty sums, the above identity also holds for $0 \leq k<n m$ and hence it works for all $k \in \mathbb{N}$. Observe that $T(k, m)=0$ when $k<n m$, since $T$ is a triangular sequence of order $n$. In particular, for $m=1$, we obtain that

$$
T(k, 1)=t_{0} \sum_{s_{1}=n}^{k}\left(\prod_{j=s_{1}}^{k-1} g(j, 1)\right) h\left(s_{1}-n, 0\right) \prod_{j=s_{0}}^{s_{1}-n-1} g(j, 0)=t_{0} \sum_{s_{1}=n}^{k}\left(\prod_{j=s_{1}}^{k-1} g(j, 1)\right) K_{n}[g, h]\left(s_{1}, 1\right),
$$

and the proposed formula is true for $m=1$. Assuming that it is true for $1 \leq j \leq m-1$, where $m \geq 2$, then

$$
\begin{aligned}
T(k, m) & =\sum_{s_{m}=m n}^{k}\left(\prod_{j=s_{m}}^{k-1} g(j, m)\right) h\left(s_{m}-n, m-1\right) T\left(s_{m}-n, m-1\right) \\
& =t_{0} \sum_{s_{m}=m n}^{k}\left(\prod_{j=s_{m}}^{k-1} g(j, m)\right) \\
& \times h\left(s_{m}-n, m-1\right) \sum_{s_{m}}^{s_{m}-n}\left(\prod_{j=s_{m-1}}^{s_{m}-1-n} g(j, m-1)\right) K_{n}[g, h]\left(s_{m-1}, m-1\right) \\
& =t_{0} \sum_{s_{m}=m n}^{k}\left(\prod_{j=s_{m}}^{k-1} g(j, m)\right) \sum_{s_{m}=m n}^{s_{m}-n} \Phi[g, h]\left(s_{m}-n, s_{m-1}\right) K_{n}[g, h]\left(s_{m-1}, m-1\right) \\
& \left.=t_{0} \sum_{j=s_{m}}^{k-1} g(j, m)\right) K_{n}[g, h]\left(s_{m}, m\right)
\end{aligned}
$$

and the result follows. The last identity is a straightforward consequence of the results of Proposition 2.2

Notice that for fixed $t_{0} \in \mathbb{A}$ and $g, h \in \ell(\mathbb{A})$ each integer $n \in \mathbb{N}^{*}$ determines a triangular sequence $T$ with order $n$. Therefore, it has sense to name $t_{0}$ the initial value and the double sequences $g$ and $h$ the generators and hence we should represent the triangular sequence of order $n$ given in the above theorem as $T=\mathscr{G}_{n}\left(t_{0}, g, h\right)$ and named it the triangular sequence of order $n$ generated by $t_{0}, g$ and $h$. However, it is also clear that $\mathscr{G}_{n}\left(t_{0}, g, h\right)=t_{0} \mathscr{G}_{n}(1, g, h)$, and hence we can restrict ourselves to the study of triangular sequences such that $T(0,0)=1$. Therefore, we denote by $\mathscr{G}_{n}(g, h)$ the unique triangular sequence of order $n$ generated by $t_{0}=1$, and $g, h \in \ell_{2}(\mathbb{A})$; that is, always $\mathscr{G}_{n}(g, h)(0,0)=1$. Besides, fixed $n \in \mathbb{N}$, we can understand $\mathscr{G}_{n}$ as a map $\mathscr{G}_{n}: \ell_{2}(\mathbb{A}) \times \ell_{2}(\mathbb{A}) \longrightarrow \Lambda_{n}(\mathbb{A})$. Observe that, with the notation of [2], $\mathscr{G}_{1}=\mathscr{G}_{\text {. In addition, fixed }}$ 
$n \in \mathbb{N}^{*}$ only the values $g(k, m)$ and $h(k, m)$ for $k \geq n m, m \geq 0$ are relevant to form the triangular sequence $\mathscr{G}_{n}(g, h)$. This remark could to lead to understand $\mathscr{G}_{n}$ as a map $\mathscr{G}_{n}: \Lambda_{n}(\mathbb{A}) \times \Lambda_{n}(\mathbb{A}) \longrightarrow \Lambda_{n}(\mathbb{A})$. However, in most of the cases, we prefer to consider the generators as arbitrary double sequences, because it permit to the same double sequences to act as generators of triangular sequences of any order.

Given $g, h \in \ell_{2}(A)$, for any $n \in \mathbb{N}^{*}$, the triangular sequence of order $n$ generated by $g$ and $h, \mathscr{G}_{n}(g, h)$, is called row-generated when $g$ and $h$ only depend on their first argument, or equivalently when there exist $\phi, \psi \in \ell(\mathbb{A})$ such that $g=\phi \otimes 1$ and $h=\psi \otimes 1$. In this case, we denote $\mathscr{G}_{n}(g, h)$ simply by $\mathscr{G}_{n}(\phi, \psi)$ and then $\phi$ and $\psi$ are called the generators of the triangular sequence. Observe that except for the Stirling numbers of second kind, all triangular sequences (i) to (iv) are row-generated. In addition, the row-generated triangular sequences will play a fundamental role in the last section of this paper.

Next, we describe some particular triangular sequences with elementary generators. In all cases the formula can be obtained straightforwardly from the above theorem.

Corollary 2.4. Given $g, h \in \ell_{2}(\mathbb{A})$ and $n \in \mathbb{N}^{*}$, the following properties are satisfied:

(i) $\mathscr{G}_{n}(g, 0)(k, m)=0$ for $m \geq 1$.

(ii) $\mathscr{G}_{n}(0, h)(k, m)=0$, for any $k, m \in \mathbb{N}, k \neq m n$. More generally, $T$ is given as above iff $g(n m, m)=0$ for any $m \in \mathbb{N}$.

(iii) Given $\alpha, \beta \in \mathbb{A}$, then $\mathscr{G}_{n}(\alpha g, \beta h)(k, m)=\alpha^{k-n m} \beta^{m} \mathscr{G}_{n}(g, h)(k, m), k \geq n m$.

(iv) $\mathscr{G}_{n}(1, h)(k, 0)=1$, for any $k \geq 0$ and

$$
\mathscr{G}_{n}(1, h)(k+m(n-1), m)=\sum_{1 \leq s_{1}<s_{2} \cdots<s_{m} \leq k} \prod_{i=0}^{m-1} h\left(s_{i+1}-1+(n-1) i, i\right), \quad k \geq m \geq 1 .
$$

(v) $\mathscr{G}_{n}(g, 1)(k, 0)=\prod_{j=0}^{k-1} g(j, 0)$, for any $k \geq 0$ and

$$
\mathscr{G}_{n}(g, 1)(k+m(n-1), m)=\sum_{1 \leq s_{1}<s_{2} \cdots<s_{m} \leq k}\left(\prod_{j=s_{m}}^{k-1} g(j+m(n-1), m)\right)\left(\prod_{i=0}^{m-1} \prod_{j=s_{i}}^{s_{i+1}-2} g(j+i(n-1), i)\right),
$$

for any $k \geq m \geq 1$.

(vi) If there exist $\phi, \psi \in \ell_{2}(\mathbb{A})$ such that $g=\phi \otimes 1$ and $h=\psi \otimes 1$, then $\mathscr{G}_{n}(\phi, \psi)(k, 0)=\phi^{\pi}(k)$ for any $k \geq 0$ and

$$
\begin{aligned}
\mathscr{G}_{n}(\phi, \psi)(k+m(n-1), m)= & \sum_{1 \leq s_{1}<\cdots<s_{m} \leq k} \\
\times & \left(\prod_{i=0}^{m-1} \psi\left(s_{i+1}-1+i(n-1)\right)\right) \\
\times & \left(\prod_{j=s_{m}}^{k-1} \phi(j+m(n-1))\right)\left(\prod_{i=0}^{m-1} \prod_{j=s_{i}}^{s_{i+1}-2} \phi(j+i(n-1))\right)
\end{aligned}
$$


As a by-product of last corollary we have the following identities for the triangular sequences of order $n=1$ determined by the recurrences (i) to (iv). If $\mathscr{G}_{1}(g, h)$ is any of this triangular sequences, we know that $\mathscr{G}_{1}(g, h)(k, 0)=\prod_{j=0}^{k-1} g(j, 0)$ for $k \geq 0$, in particular $\mathscr{G}_{1}(g, h)(0,0)=1, \mathscr{G}_{1}(g, h)(k, m)=0$ if $0 \leq k<m$ and

$$
\mathscr{G}_{1}(g, h)(k, m)=\sum_{1 \leq s_{1}<\cdots<s_{m} \leq k}\left(\prod_{i=0}^{m-1} h\left(s_{i+1}-1, i\right)\right)\left(\prod_{j=s_{m}}^{k-1} g(j, m)\right)\left(\prod_{i=0}^{m-1} \prod_{j=s_{i}}^{s_{i+1}-2} g(j, i)\right), \quad k \geq m \geq 1
$$

In particular, when $g=1$ and $h(k, m)=\frac{1}{k+1}$, we obtain that $\mathscr{G}_{1}(g, h)(k, 0)=1$, for $k \geq 0$ and moreover $\mathscr{G}_{1}(g, h)(k, m)=\sum_{1 \leq s_{1}<\cdots<s_{m} \leq k} \frac{1}{s_{1} \cdots s_{m}}$ for $k \geq m \geq 1$. Therefore, for these generators $\mathscr{G}_{1}=H$, the generalized harmonic numbers.

The remain examples, (i), (ii) and (iii) correspond to the choice $h=1$, and $g=1$ in (i), $g=\phi \otimes 1$ in (ii), or $g=1 \otimes \phi$ in (iii), which implies that

$$
\begin{aligned}
& \mathscr{G}_{1}(g, h)(k, m)=\sum_{1 \leq s_{1}<\cdots<s_{m} \leq k} 1, \quad \mathscr{G}_{1}(g, h)(k, m)=\sum_{1 \leq s_{1}<\cdots<s_{m} \leq k}\left(\prod_{j=s_{m}}^{k-1} \phi(j)\right)\left(\prod_{i=0}^{m-1} \prod_{j=s_{i}}^{s_{i+1}-2} \phi(j)\right) \\
& \text { and } \mathscr{G}_{1}(g, h)(k, m)=\sum_{1 \leq s_{1}<\cdots<s_{m} \leq k}\left(\prod_{j=s_{m}}^{k-1} \phi(m)\right)\left(\prod_{i=0}^{m-1} \prod_{j=s_{i}}^{s_{i+1}-2} \phi(i)\right), \quad k \geq m \geq 1, \quad \text { respectively. }
\end{aligned}
$$

(i) Binomial coefficients: In this case $B=\mathscr{G}_{1}(1,1)$. Since $\mathscr{G}_{1}(1,1)(k, 0)=1$ for any $k \geq 0$ and for any $k \geq m \geq 1$ we have that

$$
B(k, m)=\sum_{1 \leq s_{1}<s_{2} \cdots<s_{m} \leq k} 1=\left(\begin{array}{c}
k \\
m
\end{array}\right),
$$

we obtain that $B(k, m)=\left(\begin{array}{c}k \\ m\end{array}\right)$ for any $k \geq m \geq 0$.

(ii) Signless Stirling numbers of first kind: In this case, $\left[\begin{array}{l}k \\ m\end{array}\right]=\mathscr{G}_{1}(g, 1)$ where $g(k, m)=k, k, m \in \mathbb{N}$. Therefore, $\left[\begin{array}{l}k \\ 0\end{array}\right]=0$ for $k \geq 1$ and moreover, since $g(0, m)=0$ for any $m \in \mathbb{N}$,

$$
\begin{aligned}
{\left[\begin{array}{l}
k \\
m
\end{array}\right] } & =\sum_{1 \leq s_{1}<\cdots<s_{m} \leq k}\left(\prod_{j=s_{m}}^{k-1} j\right)\left(\prod_{i=0}^{m-1} \prod_{j=s_{i}}^{s_{i+1}-2} j\right)=\sum_{1=s_{1}<\cdots<s_{m} \leq k} \frac{(k-1) !}{\left(s_{m}-1\right) !} \prod_{i=1}^{m-1} \frac{\left(s_{i+1}-2\right) !}{\left(s_{i}-1\right) !} \\
& =\sum_{1<s_{2}<\cdots<s_{m} \leq k} \frac{(k-1) !}{\left(s_{m}-1\right) \cdots\left(s_{2}-1\right)}=\left(\begin{array}{c}
(k-1) ! \sum_{1 \leq s_{2}<\cdots<s_{m} \leq k-1} \\
\frac{1}{s_{m} \cdots s_{2}}
\end{array}=(k-1) ! H(k-1, m-1)\right.
\end{aligned}
$$

a known equality obtained by V. Adamchik in [5]. 
(iii) Stirling numbers of second kind: In this case, $\left\{\begin{array}{l}k \\ m\end{array}\right\}=\mathscr{G}_{1}(g, 1)$ where $g(k, m)=m, k, m \in \mathbb{N}$. Therefore, $\left\{\begin{array}{l}k \\ 0\end{array}\right\}=0$ for $k \geq 1$ and moreover, since $g(k, 0)=0$ for any $k \geq m \geq 1$ we have that

$$
\begin{aligned}
\left\{\begin{array}{c}
k \\
m
\end{array}\right\} & =\sum_{1 \leq s_{1}<\cdots<s_{m} \leq k}\left(\prod_{j=s_{m}}^{k-1} m\right)\left(\prod_{i=0}^{m-1} \prod_{j=s_{i}}^{s_{i+1}-2} i\right)=\sum_{1=s_{1}<\cdots<s_{m} \leq k} m^{k-s_{m}} \prod_{i=1}^{m-1} i^{s_{i+1}-s_{i}-1} \\
& =\sum_{s_{m}=m}^{k} m^{k-s_{m}} \sum_{s_{m-1}=m-1}^{s_{m}-1}(m-1)^{s_{m}-s_{m-1}-1} \cdots \sum_{s_{3}=3}^{s_{4}-1} 3^{s_{4}-s_{3}-1} \sum_{s_{2}=2}^{s_{3}-1} 2^{s_{3}-s_{2}-1} \\
& =\frac{1}{m !} \sum_{j=0}^{m-1}(-1)^{j}\left(\begin{array}{c}
m \\
j
\end{array}\right)(m-j)^{k}=\frac{1}{m !} \sum_{j=0}^{m}(-1)^{m-j}\left(\begin{array}{c}
m \\
j
\end{array}\right) j^{k}
\end{aligned}
$$

where we have repeatedly applied the identity

$$
\sum_{j=s}^{r} \frac{s^{r-j}}{(s-1) !} \sum_{i=0}^{s-1}(-1)^{s-1-i}\left(\begin{array}{c}
s-1 \\
i
\end{array}\right) i^{j-1}=\frac{1}{s !} \sum_{i=0}^{r}(-1)^{s-i}\left(\begin{array}{c}
s \\
i
\end{array}\right) i^{r}, \quad 2 \leq s \leq r .
$$

We end this section with some well-known results about the original question by Graham, Knuth and Patashnik on triangular sequences of order 1 with affine generators. Specifically, using a sort of mixture between the notation in [2] and in [10], given $a_{0}, a_{1}, b_{0}, b_{1}, c_{0}, c_{1} \in \mathbb{A}$ we denote

$$
\mathscr{G}\left(a_{0}, b_{0}, c_{0} ; a_{1}, b_{1}, c_{1}\right)=\mathscr{G}_{1}(g, h), \text { where } g(k, m)=a_{0} k+b_{0} m+c_{0}, h(k, m)=a_{1} k+b_{1} m+c_{1} .
$$

According with Theorem 2.3 we have that $\mathscr{G}\left(a_{0}, b_{0}, c_{0} ; a_{1}, b_{1}, c_{1}\right)(k, 0)=\prod_{j=0}^{k-1}\left(a_{0} j+c_{0}\right)$ for $k \geq 0$ and

$$
\begin{aligned}
\mathscr{G}\left(a_{0}, b_{0}, c_{0} ; a_{1}, b_{1}, c_{1}\right)(k, m)=\sum_{1 \leq s_{1}<\cdots<s_{m} \leq k} & \left(\prod_{i=1}^{m}\left(a_{1} s_{i}+b_{1} i+c_{1}-a_{1}-b_{1}\right)\right) \\
& \times\left(\prod_{j=s_{m}}^{k-1}\left(a_{0} j+b_{0} m+c_{0}\right)\right)\left(\prod_{i=0}^{m-1} \prod_{j=s_{i}}^{s_{i+1}-2}\left(a_{0} j+b_{0} i+c_{0}\right)\right),
\end{aligned}
$$

for any $k \geq m \geq 1$.

Barbero, Salas and Villaseñor classified this kind of triangular sequences, or special hyperbinomials sequences in Théorêt's terminology, according the values of $b_{0}$ and $b_{1}$ that are the fundamental parameters. They are named them of Type $I$ when $b_{0} b_{1} \neq 0$, of Type $I I$ when $b_{0} \neq 0$ and $b_{1}=0$, of Type III when $b_{0}=0$ and $b_{1} \neq 0$ and of Type $I V$ when $b_{0}=b_{1}=0$.

We remark that the formulas given in 7 for the special hyperbinomials sequences are expressed implicitly in terms of bivariate exponential generating functions, from which, in general, it is very difficult to obtain 
explicit identities for such sequences. In fact, this can be made in precisely those cases that were previously obtained by several authors. Next, we give a summary of the available formulas, referring each of it to the work where was obtained, not necessarily for the first time.

Proposition 2.5. Given $a_{0}, a_{1}, b_{0}, b_{1}, c_{0}, c_{1} \in \mathbb{A}$, for any $k, m \in \mathbb{N}$ such that $0 \leq k \leq n$, the following identities are satisfied:

(i) [8, Theorem 6] and [7, Corollary 4.5]:

$$
\mathscr{G}\left(a_{0}, 0, c_{0} ; a_{1}, 0, c_{1}\right)(k, m)=\sum_{s_{2}=0}^{k} \sum_{s_{1}=0}^{m}\left[\begin{array}{l}
k \\
s_{2}
\end{array}\right]\left(\begin{array}{c}
s_{2} \\
s_{1}
\end{array}\right)\left(\begin{array}{c}
k-s_{2} \\
m-s_{1}
\end{array}\right) a_{0}^{k-s_{2}+s_{1}-m} a_{1}^{m-s_{1}} c_{0}^{s_{2}-s_{1}} c_{1}^{s_{1}}
$$

(ii) [2, Theorem 18] and [8, Theorem 4]:

$$
\mathscr{G}\left(a_{0}, b_{0}, c_{0} ; 0, b_{1}, c_{1}\right)(k, m)=\left(\prod_{j=0}^{m-1}\left(b_{1} j+c_{1}\right)\right) \sum_{s_{2}=0}^{k} \sum_{s_{1}=0}^{k}\left[\begin{array}{l}
k \\
s_{2}
\end{array}\right]\left(\begin{array}{l}
s_{2} \\
s_{1}
\end{array}\right)\left\{\begin{array}{l}
s_{1} \\
m
\end{array}\right\} a_{0}^{k-s_{2}} b_{0}^{s_{1}-m} c_{0}^{s_{2}-s_{1}}
$$

(iii) [8, Theorem 5]:

$$
\begin{aligned}
\mathscr{G}\left(a_{0},-a_{0}, c_{0} ; a_{1}, b_{1}, c_{1}\right)(k, m)=\left(\prod_{j=0}^{k-m-1}\left(a_{0} j+c_{0}\right)\right) \sum_{s_{2}=0}^{k} \sum_{s_{1}=0}^{k}\left[\begin{array}{l}
k \\
s_{2}
\end{array}\right]\left(\begin{array}{c}
s_{2} \\
s_{1}
\end{array}\right)\left\{\begin{array}{c}
s_{1} \\
k-m
\end{array}\right\} \\
\times\left(a_{1}+b_{1}\right)^{k-s_{2}}\left(-b_{1}\right)^{s_{1}-k+m} c_{1}^{s_{2}-s_{1}} .
\end{aligned}
$$

(iv) [8, Theorem 8]: If $b_{0} b_{1} \neq 0$ and $\frac{a_{0}}{b_{0}}=1+\frac{a_{1}}{b_{1}}$,

$$
\begin{aligned}
\mathscr{G}\left(a_{0}, b_{0}, c_{0} ; a_{1}, b_{1}, c_{1}\right)(k, m)=\sum_{\ell=0}^{k} & \left(\prod_{j=1}^{k-\ell}\left(1-j+\frac{c_{1}}{b_{1}}-\frac{c_{0}}{b_{0}}\right)\right)\left(\begin{array}{c}
\ell \\
m
\end{array}\right) \\
& \times \sum_{s_{2}=0}^{k} \sum_{s_{1}=0}^{k}\left[\begin{array}{c}
k \\
s_{2}
\end{array}\right]\left(\begin{array}{c}
s_{2} \\
s_{1}
\end{array}\right)\left\{\begin{array}{c}
s_{1} \\
k-\ell
\end{array}\right\}(-1)^{s_{1}} a_{0}^{k-s_{2}} b_{0}^{s_{2}-m} b_{1}^{s_{1}-s_{2}+m} c_{1}^{s_{2}-s_{1}} .
\end{aligned}
$$

\section{Row-generated triangular sequences and three-term recurrences}

The main motivation of this work was to obtain a closed expression of the solution of homogeneous three-term sequences in terms of their coefficients. The relation between the solutions and double sequences determined by a recurrence as (11) leaded us to analyze triangular sequences of order greater than 1 . A preliminary study, focused on second order linear difference equations, and hence on triangular sequences of order 2 , can be found in 13 .

Given sequences $a \in \ell\left(\mathbb{A}^{*}\right)$ and $b, c \in \ell(\mathbb{A})$, for any $n \in \mathbb{N}^{*}$, we consider the homogeneous three-term recurrence of order $n$

$$
a(k+2-n) z(k+1)-b(k) z(k)-c(k+1-n) z(k+1-n)=0, \quad k \geq n-1 .
$$


The equation is named explicit when $a=1$. It is clear that the above identity determines a difference equation of order $n$ with $a, b$ and $c$ as its coefficients. In particular, when $n=1$, the difference equation (14) becomes

$$
z(k+1)=\hat{a}(k) z(k), \quad k \geq 0,
$$

where $\hat{a}(k)=a(k+1)^{-1}(b(k)+c(k))$ for any $k \in \mathbb{N}$, whereas when $n=2$ we have the usual three-term recurrence of second order

$$
a(k) z(k+1)-b(k) z(k)-c(k-1) z(k-1)=0, \quad k \geq 1 .
$$

A complete treatment of second order linear difference equations, homogeneous or not, can be found in [1].

Notice that the values $a(0)$ and $b(k), 0 \leq k<n-1$ are not relevant to solve the recurrence (14), and hence they can be chosen arbitrarily. Therefore, we can always assume without lose of generality that $a(0)=1$, which implies that $a^{\pi}(k)=1$ for any $k \leq 1$. Moreover, since $a(k) \in \mathbb{A}^{*}$ it is easy to conclude that given $q_{j} \in \mathbb{A}, j=0, \ldots, n-1$, the initial value problem

$$
a(k+2-n) z(k+1)-b(k) z(k)-c(k+1-n) z(k+1-n)=0, \quad k \geq n-1, \quad z(j)=q_{j}, \quad j=0, \ldots, n-1,
$$

has a unique solution.

The relation between row-generated triangular sequences and three-term recurrences is given in the following result.

Theorem 3.1. Given $a \in \ell\left(\mathbb{A}^{*}\right)$ and $b, c \in \ell(\mathbb{A})$ then, the unique solution of the initial value problem $a(k+2-n) z(k+1)-b(k) z(k)-c(k+1-n) z(k+1-n)=0, \quad k \geq n-1, \quad z(i)=b^{\pi}(i), \quad i=0, \ldots, n-1$, is given by

$$
z(k)=a^{\pi}(k+2-n)^{-1} \sum_{m=0}^{\left\lfloor\frac{k}{n}\right\rfloor} \mathscr{G}_{n}\left(b, a \star_{n} c\right)(k, m), \quad k \in \mathbb{N} .
$$

More explicitly,

$$
\begin{aligned}
z(k)=a^{\pi}(k+2-n)^{-1}\left[b^{\pi}(k)+\sum_{m=1}^{\left\lfloor\frac{k}{n}\right\rfloor} \sum_{1 \leq s_{1}<\cdots<s_{m} \leq k-m(n-1)}\right. & \left(\prod_{i=0}^{m-1}\left(a \star_{n} c\right)\left(s_{i+1}-1+i(n-1)\right)\right) \\
& \times\left(\prod_{j=s_{m}}^{k-m(n-1)-1} b(j+m(n-1))\right) \\
& \left.\times\left(\prod_{i=0}^{m-1} \prod_{j=s_{i}}^{s_{i+1}-2} b(j+i(n-1))\right)\right],
\end{aligned}
$$

for any $k \in \mathbb{N}$. 
Proof. Let $T=\mathscr{G}_{n}\left(b, a \star_{n} c\right)$ and consider the sequence $z \in \ell(\mathbb{A})$ determined by the identity

$$
z(k)=a^{\pi}(k+2-n)^{-1} \sum_{m=0}^{\left\lfloor\frac{k}{n}\right\rfloor} T(k, m), k \in \mathbb{N} .
$$

If $0 \leq k<n$ we have that $a^{\pi}(k+2-n)=1,\left\lfloor\frac{k}{n}\right\rfloor=0$ and hence,

$$
\begin{gathered}
z(k)=T(k, 0)=b^{\pi}(k), \quad k=0, \ldots, n-1 . \\
z(n)=a(0)^{-1} a(1)^{-1}\left[b^{\pi}(n)+\left(a \star_{n} c\right)(0)\right] \\
a(1) z(n)-b(n-1) z(n-1)-c(0) z(0)=a(1) 0-b^{\pi}(n)-c(0)
\end{gathered}
$$

On the other hand, if $k \geq n-1$, then

$$
\begin{aligned}
a(k+2-n) z(k+1) & =a^{\pi}(k+2-n)^{-1} \sum_{m=0}^{\left\lfloor\frac{k+1}{n}\right\rfloor} T(k+1, m) \\
& =a^{\pi}(k+2-n)^{-1} \sum_{m=0}^{\left\lfloor\frac{k+1}{n}\right\rfloor}\left[b(k) T(k, m)+\left(a \star_{n} c\right)(k+1-n) T(k+1-n, m-1)\right] .
\end{aligned}
$$

Consider $\ell \in \mathbb{N}^{*}$ and $0 \leq r<n$ such that $k=\ell n+r$. If $0 \leq r<n-1$, then $\left\lfloor\frac{k+1}{n}\right\rfloor=\left\lfloor\frac{k}{n}\right\rfloor=\ell$, whereas, if $r=n-1$, then $\left\lfloor\frac{k+1}{n}\right\rfloor=1+\left\lfloor\frac{k}{n}\right\rfloor=1+\ell$, which implies that $T\left(k,\left\lfloor\frac{k+1}{n}\right\rfloor\right)=0$. Therefore, in both cases,

$$
\begin{aligned}
a(k+2-n) z(k+1) & =b(k) z(k)+\left(a \star_{n} c\right)(k+1-n) a^{\pi}(k+2-n)^{-1} \sum_{m=1}^{\left\lfloor\frac{k+1}{n}\right\rfloor} T(k+1-n, m-1) \\
& =b(k) z(k)+c(k+1-n) a^{\pi}(k+3-2 n)^{-1} \sum_{m=0}^{\left\lfloor\frac{k+1-n}{n}\right\rfloor} T(k+1-n, m) \\
& =b(k) z(k)+c(k+1-n) z(k+1-n) .
\end{aligned}
$$

Observe that in the above Theorem, we always have the initial condition $z(0)=1$. Besides, if given $n \geq 2$ we consider $q_{0}, \ldots, q_{n-1}$ such that $q_{0}=1$ and $q_{j} \in \mathbb{A}^{*}$ for $1 \leq j \leq n-2$, we can assume without lose of generality that

$$
b(j)=q_{j}^{-1} q_{j+1}, \text { for any } j=0, \ldots, n-2 .
$$

since $b(j), j=0, \ldots, n-2$ can be chosen arbitrarily. Then, the initial value problem 15 coincides with the initial value problem in which the initial conditions are $z(i)=b^{\pi}(i), i=0, \ldots, n-1$.

We remark that the unique solution of the initial value problem in the above theorem can be also expressed as

$$
z(k)=a^{\pi}(k+2-n)^{-1} \sum_{m=0}^{k} \mathscr{G}_{n}\left(b, a \star_{n} c\right)(k, m)=a^{\pi}(k+2-n)^{-1} \sum_{m=0}^{\infty} \mathscr{G}_{n}\left(b, a \star_{n} c\right)(k, m), \quad k \in \mathbb{N},
$$


since $\mathscr{G}\left(b, a \star_{n} c\right)$ is a triangular sequence of order $n$. Next, we give the expression of the solution in the particular case when the equation (14) is explicit.

Corollary 3.2. Given $b, c \in \ell(\mathbb{A})$, for any $n \in \mathbb{N}^{*}$, the unique solution of the initial value problem

$$
z(k+1)-b(k) z(k)-c(k+1-n) z(k+1-n)=0, \quad k \geq n-1, \quad z(i)=b^{\pi}(i), \quad i=0, \ldots, n-1,
$$

is given by

$$
\begin{aligned}
z(k) & =\sum_{m=0}^{\left\lfloor\frac{k}{n}\right\rfloor} \mathscr{G}_{n}(b, c)(k, m)=\sum_{m=0}^{\infty} \mathscr{G}_{n}(b, c)(k, m) \\
= & b^{\pi}(k)+\sum_{m=1}^{\left\lfloor\frac{k}{n}\right\rfloor} \sum_{1 \leq s_{1}<\cdots<s_{m} \leq k-m(n-1)}\left(\prod_{i=0}^{m-1} c\left(s_{i+1}-1+i(n-1)\right)\right) \\
& \times\left(\prod_{j=s_{m}}^{k-m(n-1)-1} b(j+m(n-1))\right)\left(\prod_{i=0}^{m-1} \prod_{j=s_{i}}^{s_{i+1}-2} b(j+i(n-1))\right),
\end{aligned}
$$

for any $k \in \mathbb{N}$. In particular, when $n=1$ we have

$$
\sum_{m=0}^{\infty} \mathscr{G}_{1}(b, c)(k, m)(k, m)=\prod_{s=0}^{k-1}(b(s)+c(s)) \quad \text { and } \quad \sum_{m=0}^{\infty}(-1)^{m} \mathscr{G}_{1}(b, c)(k, m)=\prod_{s=0}^{k-1}(b(s)-c(s)), k \in \mathbb{N} .
$$

We remark that the above corollary allows us to determine the sums of each row in a a row-generated infinite matrix; that is, a triangular sequence whose generators depending only on its first argument. This specific result when $n=1$, appeared in a slightly general form in [2, Theorem 17]. In fact, it is easy to modify the reasoning in the above Theorem to prove that the result is still valid for triangular sequences whose generators $g$ and $h$ satisfy that $g(k, m)+h(k, m-1)$ does not depend on $m$. Many interesting combinatorial identities can be obtained from this simple result, see for instance [2, 8 .

As a nice example of the application of the above results, we consider the following initial value problem, which solution we name Fibonacci numbers of order $n \in \mathbb{N}^{*}$ :

$$
f(k+1)=f(k)+f(k+1-n), \quad f_{0}=\cdots=f_{n-1}=1, \quad k \geq n-1 .
$$

Notice that the Fibonacci numbers of order 2 are the well-known Fibonacci numbers. From the above Corollary, we obtain that

$$
f_{k}=1+\sum_{m=1}^{\left\lfloor\frac{k}{n}\right\rfloor} \sum_{1 \leq s_{1}<\cdots<s_{m} \leq k-m(n-1)} 1=\sum_{m=0}^{\left\lfloor\frac{k}{n}\right\rfloor}\left(\begin{array}{c}
k-m(n-1) \\
m
\end{array}\right), \quad k \in \mathbb{N} .
$$

Therefore, for the standard Fibonacci numbers we have the well-known Lucas Formula $f_{k}=\sum_{m=0}^{\left\lfloor\frac{k}{2}\right\rfloor}\left(\begin{array}{c}k-m \\ m\end{array}\right)$, $k \in \mathbb{N}$, see for instance [14, Theorem 12.4]. In addition, for $n=1$ we also obtain the well-known binomial identities

$$
f_{k}=\sum_{m=0}^{k}\left(\begin{array}{c}
k \\
m
\end{array}\right)=2^{k} \text { and } \sum_{m=0}^{k}(-1)^{m}\left(\begin{array}{c}
k \\
m
\end{array}\right)=0, \quad k \in \mathbb{N} .
$$


Fibonacci numbers of order $n$ appears as a very specific case of the three-term recurrence with constant coefficients, that among the initial value problems of the type $[15$, are the most easy-to-solve.

Corollary 3.3. Given $b, c \in \mathbb{A}$, then the unique solution of the initial value problem

$$
z(k+1)-b z(k)-c z(k+1-n)=0, \quad z(i)=b^{i}, \quad i=0, \ldots, n-1,
$$

is given by

$$
z(k)=\sum_{m=0}^{\left\lfloor\frac{k}{n}\right\rfloor} b^{k-m n} c^{m}\left(\begin{array}{c}
k-m(n-1) \\
m
\end{array}\right), \quad k \in \mathbb{N} .
$$

In particular, when $\mathbb{A}$ is an integral domain, $b c \neq 0$, and $r$ is the solution of the equation $r^{n}=c$ in the algebraic closure of the field of fractions of $\mathbb{A}$, we obtain that,

$$
z(k)=r^{k} \sum_{m=0}^{\left\lfloor\frac{k}{n}\right\rfloor}\left(\frac{b}{r}\right)^{k-n m}\left(\begin{array}{c}
k-(n-1) m \\
m
\end{array}\right), \quad k \in \mathbb{N} .
$$

We remark the advantage of our method with respect to the classical one, leading to the so-called Binet Formulae, that is based on the roots of the polynomial $p(x)=x^{n}-b x^{n-1}-c \in \mathbb{A}[x]$.

A less trivial example is given by the so-called Motzkin sequence that is the solution of the homogeneous linear second order equation in $\mathbb{Q}$

$$
(k+3) M(k+1)-(2 k+3) M(k)-3 k M(k-1)=0, \quad M(0)=M(1)=1 .
$$

Moztkin sequence appears related with many combinatorial problems, see for instance [15, 16] for the origin and the solution of the above equation. It is well-know that

$$
M(k)=\sum_{m=0}^{\left\lfloor\frac{k}{2}\right\rfloor} \frac{1}{m+1}\left(\begin{array}{c}
k \\
2 m
\end{array}\right)\left(\begin{array}{c}
2 m \\
m
\end{array}\right), \quad k \in \mathbb{N},
$$

see [16, Identity (3)]. Using the formula in Theorem 3.1 for $n=2$

$$
z(k)=\left(\prod_{j=1}^{k-1} a(j)\right)^{-1} \sum_{m=0}^{\left\lfloor\frac{k}{2}\right\rfloor} \mathscr{G}_{2}(b, a c)(k, m), k \in \mathbb{N},
$$

leads after some calculus to the following identity

$$
M(k)=\frac{2^{k+1}}{(k+1)(k+2)} \sum_{m=0}^{\left\lfloor\frac{k}{2}\right\rfloor}\left(\frac{3}{4}\right)^{m} \frac{\left(\frac{3}{2}\right)_{k-m}}{(k-2 m) ! m !}, \quad k \in \mathbb{N},
$$

see [13], that seem to be new.

We end this paper presenting some particular cases of Theorem 3.1 that recover many classical solutions of both difference and differential linear equations of second order. First we show a general framework and then we specialize the results. 
Corollary 3.4. Consider $r, x \in \mathbb{A}, a \in \ell\left(\mathbb{A}^{*}\right)$ and $b, c \in \ell(\mathbb{A})$. Then, the unique solution of the initial value problem

$$
\begin{array}{rlrl}
0 & =a(k+2-n) z(k+1)-r x b(k) z(k)-c(k+1-n) z(k+1-n), & & k \geq n-1 \\
z(i) & =(r x)^{i} b^{\pi}(i), & i=0, \ldots, n-1,
\end{array}
$$

is given by

$$
z(k)=P_{k}(x)=a^{\pi}(k+2-n)^{-1} \sum_{m=0}^{\left\lfloor\frac{k}{n}\right\rfloor}(r x)^{k-n m^{2}} \mathscr{G}_{n}\left(b, a \star_{n} c\right)(k, m), \quad k \in \mathbb{N},
$$

and hence, for any $k \in \mathbb{N}, P_{k} \in \mathbb{A}[x]$ has degree less than or equal to $k$. Moreover, when $n$ is even, then $P_{k}(-x)=(-1)^{k} P_{k}(x)$ for any $k \in \mathbb{N}$.

We finish by showing some classical examples for difference equations of second order that lead to orthogonal polynomials. By the way, our methodology explain the, in some sense, mysterious fact that in the expression of these families of polynomials the sum is extended up $\left\lfloor\frac{n}{2}\right\rfloor$.

(i) Fibonacci equation: $f(k+1)-x f(k)-f(k-1)=0, \quad k \leq 1, \quad f(0)=1, f(1)=x$. The solution is given by the so-called $k$-th Fibonacci polynomials

$$
F_{k}(x)=\sum_{m=0}^{\left\lfloor\frac{k}{2}\right\rfloor} x^{k-2 m}\left(\begin{array}{c}
k-m \\
m
\end{array}\right), \quad k \in \mathbb{N} .
$$

(ii) Chebyshev equation: $u(k+1)-2 x u(k)+u(k-1)=0, \quad k \leq 1, \quad u(0)=1, u(1)=2 x$. The solution is given by the so-called $k$-th Chebyshev polynomials of second kind, see [17,

$$
U_{k}(x)=\sum_{m=0}^{\left\lfloor\frac{k}{2}\right\rfloor}(-1)^{m}(2 x)^{k-2 m}\left(\begin{array}{c}
k-m \\
m
\end{array}\right), \quad k \in \mathbb{N} .
$$

(iii) Hermite equation: $h(k+1)-2 q x h(k)+2 q(k+1) h(k-1)=0, \quad k \leq 1, \quad h(0)=1, h(1)=2 x q, q \in \mathbb{A}$. The solution is given by the so-called $k$-th Hermite polynomials of second kind, see [3],

$$
H_{k}(x)=\sum_{m=0}^{\left\lfloor\frac{k}{2}\right\rfloor}(-1)^{m} q^{k-m}(2 x)^{k-2 m} \frac{k !}{m !(k-2 m) !}, \quad k \in \mathbb{N} .
$$

(iv) Gegenbauer equation: $(k+1) g(k+1)-2 x(k+q) g(k)+r(k+2 q) g(k-1)=0, \quad k \leq 1, \quad g(0)=1, g(1)=$ $2 x q, q \in \mathbb{A}$. The solution is given by the so-called $k$-th Gegenbauer polynomials of second kind, see [3],

$$
G_{k}(x)=\sum_{m=0}^{\left\lfloor\frac{k}{2}\right\rfloor}(-r)^{m}(2 x)^{k-2 m} \frac{1}{m !(k-2 m) !}(q)_{k-m}, \quad k \in \mathbb{N} .
$$

Notice that the choice $q=\frac{1}{2}$ leads to the Legendre equation and hence to the Legendre polynomials.

This work has been partly supported by the Spanish Program I+D+i (Ministerio de Economía y Competitividad) under projects MTM2014-60450-R, MTM2017-85996-R and MTM2017-90682-REDT. 


\section{References}

220 [1] A. M. Encinas, M. J. Jiménez, Second order linear difference equations, J. Differ. Equ. Appl. 24 (3) (2018) $305-343$. doi:10.1080/10236198.2017.1408608

[2] E. Neuwirth, Recursively defined combinatorial functions: extending Galton's board, Discrete Math. 239 (2001) $35-51$. doi:10.1016/S0012-365X(00)00373-3

[3] E. D. Rainville, Special Functions, Chelsea Publishing Company, New York, 1960.

225 [4] I. M. Gessel, On Miki's identity for Bernoulli numbers, J. Number Theory 110 (2005) 75-82. doi:j.jnt.2003.08.010

[ [5] V. Adamchik, On Stirling numbers and Euler sums, J. Comput. Appl. Math. 79 (1997) 119-130. doi:10.1016/ S0377-0427(96)00167-7.

[6] G. S. Cheon, M. E. A. El-Mikkawy, Generalizad harmonic numbers with riordan arrays, J. Number Theory 128 (2008) 413-425. doi:10.1016/j.jnt.2007.08.011

[7] J. F. Barbero, J. Salas, E. J. S. V. nor, Bivariate generating functions for a class of linear recurrences: general structure, J. Combin. Theory Ser. A 125 (2014) 146-165. doi:10.1016/j.jcta.2014.02.007

[8] M. Z. Spivey, On solutions to a general combinatorial recurrence, J. Integer Seq. 14 (2011) Article 11.9 .7$.

[9] R. L. Graham, D. E. Knuth, O. Patashnik, Concrete Mathematics, 2nd Edition, Addison-Wesley, Reading, MA, 1994.

[10] P. Théorêt, Fonctions génératrices pour une classe d'équations aux différences partielles, Ann. Sci. Math. Québec 19 (1995) 91-105.

[11] P. Théorêt, Relations matricielles pour hyperbinomiales, Ann. Sci. Math. Québec 19 (1995) 197-212.

[12] P. Théorêt, Hyperbinomiales multiples, Ann. Sci. Math. Québec 21 (1997) 167-176.

[13] M. J. Jiménez, A. M. Encinas, Combinatorial recurrences and linear difference equations, Electron. Notes Discrete Math. 54 (2016) 313-318. doi:j.endm.2016.09.054

[14] T. Koshy, Fibonacci and Lucas numbers with applications, Wiley, New York, 2001.

[15] F. R. Bernhart, Catalan, Motzkin and Riordan numbers, Discrete Math. 204 (1999) 73-112. doi:10.1016/S0012-365X(99) 00054-0

[16] R. Donaghey, L. W. Shapiro, Motzkin numbers, J. Combin. Theory, Ser. A 23 (1977) 291-301. doi:10.1016/0097-3165(77) 90020-6

245 [17] J. C. Mason, D. C. Handscomb, Chebyshev Polynomials, Chapman \& Hall/CRC, 2003. 